\title{
Fresnel analysis of wave propagation in nonlinear electrodynamics
}

\author{
Yuri N. Obukhov* \\ Instituto de Física Teórica, UNESP, Rua Pamplona 145, 01405-900 São Paulo, SP, Brazil \\ Guillermo F. Rubilar \\ Institute for Theoretical Physics, University of Cologne, 50923 Köln, Germany
}

(Received 11 April 2002; published 31 July 2002)

\begin{abstract}
We study wave propagation in local nonlinear electrodynamical models. Particular attention is paid to the derivation and the analysis of the Fresnel equation for the wave covectors. For the class of local nonlinear Lagrangian nondispersive models, we demonstrate how the originally quartic Fresnel equation factorizes, yielding the generic birefringence effect. We show that the closure of the effective constitutive (or jump) tensor is necessary and sufficient for the absence of birefringence, i.e., for the existence of a unique light cone structure. As another application of the Fresnel approach, we analyze the light propagation in a moving isotropic nonlinear medium. The corresponding effective constitutive tensor contains nontrivial skewon and axion pieces. For nonmagnetic matter, we find that birefringence is induced by the nonlinearity, and derive the corresponding optical metrics.
\end{abstract}

DOI: 10.1103/PhysRevD.66.024042

PACS number(s): 04.20.Cv, 04.30.Nk, 11.10.Lm

\section{INTRODUCTION}

Wave phenomena belong to the most interesting and important processes in physics. Among other field theories, nonlinear electrodynamics attracts a lot of attention in connection with the prominent role played by light in the experimental and theoretical studies of the structure of spacetime and matter.

Nonlinearities in electrodynamical models can arise in different ways in classical and quantum field theories. For example, the old Born-Infeld theory [1] was a fundamental theory alternative to classical Maxwell electrodynamics which provided a model of a classical electron. On the other hand, quantum Maxwell electrodynamics predicts nonlinear effects which arise due to radiative corrections, see [2-4]. Finally, in modern string theories a generalized Born-Infeld action naturally arises as the leading part of the effective string action, see [5-7], for example.

Wave propagation in the various nonlinear electrodynamical theories was studied previously in [8-13] and also in [14-19]. A general feature revealed in these studies is the existence of birefringence. In crystal optics, the notion of birefringence means the emergence of two rays (ordinary and extraordinary) with different velocities inside the material medium. We will use the expression "birefringence" in a similar sense, associating it with the situation when two different light cones exist for the wave normal covectors. However, the earlier results are incomplete in the sense that the full Fresnel equation, governing the wave normals, was never derived explicitly. Moreover, it was not demonstrated how it happens that the original quartic surface of wave normals reduces to the light cone. That is the primary interest in our study, and we will try to clarify this aspect for local nonlinear electrodynamical models, using and expanding our

\footnotetext{
*On leave from Department of Theoretical Physics, Moscow State University, 117234 Moscow, Russia.
}

earlier results obtained within the framework of linear electrodynamics [20,21].

We restrict our attention to local electrodynamical models, thus assuming that, at each point, the corresponding (effective) constitutive tensor of the medium under consideration depends only on the field strength itself and not on its derivatives. In other words, dispersive effects are neglected. This approximation is valid in general when the wavelength of the propagating electromagnetic field is larger than the length scales related to the microscopic (sub)structure of the medium. For instance, for the Heisenberg-Euler effective Lagrangian, which describes nonlinearities due to radiative corrections in QED, this means that the wavelength of the photons has to be much larger than the Compton length of the electron. For the isotropic media considered in Sec. XI, this means that the wavelength of the photons is assumed to be much larger than the length scales related to the resonance frequencies of the medium.

Our basic tool will be the general formula for the Fresnel equation derived earlier within linear electrodynamics $[20,21]$. Now we observe that the analysis of the wave propagation in a general local nonlinear model reduces to the linear case because the jumps of the derivatives of the excitation and of the field strength are in all cases related by a linear law. We can then make use of our master formula for the Fresnel tensor and derive the Fresnel equation for any local nonlinear model, and thereby explain the reduction to the light cones.

\section{ELECTROMAGNETIC WAVES AND FRESNEL TENSOR}

Quite generally, Maxwell's equations for the excitation 2-form $H=(\mathcal{D}, \mathcal{H})$ and the field strength 2-form $F=(E, B)$ read

$$
d H=J, \quad d F=0 .
$$


Here $J$ is the electric current 3 -form. These equations must be supplemented by a constitutive law $H=H(F)$. The latter relation contains the crucial information about the underlying physical continuum (i.e., spacetime and/or material medium). Mathematically, this constitutive law arises either from a suitable phenomenological theory of a medium or from the electromagnetic field Lagrangian. It can be a nonlinear or even nonlocal relation between the electromagnetic excitation and the field strength.

If local coordinates $x^{i}$ are given, with $i, j, \ldots=0,1,2,3$, we can decompose the excitation and field strength 2-forms into their components according to

$$
H=\frac{1}{2} H_{i j} d x^{i} \bigwedge d x^{j}, \quad F=\frac{1}{2} F_{i j} d x^{i} \bigwedge d x^{j} .
$$

There are several approaches to the study of the wave propagation in nonlinear electrodynamics. For example, one can consider the geometric optics approximation scheme when a field strength is split into a sum of a background field plus a wave term of the form $f_{i j} e^{i \Phi}$ where the amplitude $f$ varies slowly compared to the phase $\Phi$. Under the condition that the scale over which the background electromagnetic field varies is much larger than that of variations of $\Phi$, one then derives the algebraic system for the amplitude $f$ which ultimately determines the characteristics of the wave solutions in terms of the background field. The advantage of this approach is its clear physical interpretation.

An alternative approach is represented by the Hadamard theory of weak discontinuities. It yields the same results, whereas it is more mathematically transparent, in our opinion. Correspondingly, here we will study the propagation of a discontinuity of the electromagnetic field using the Hadamard approach following the lines of Ref. [20], see also Ref. [21]. The surface of discontinuity $S$ is defined locally by a function $\Phi$ such that $\Phi=$ const on $S$. Across $S$, the geometric Hadamard conditions are satisfied:

$$
\begin{aligned}
& {\left[F_{i j}\right]=0, \quad\left[\partial_{i} F_{j k}\right]=q_{i} f_{j k},} \\
& {\left[H_{i j}\right]=0, \quad\left[\partial_{i} H_{j k}\right]=q_{i} h_{j k} .}
\end{aligned}
$$

Here $[\mathcal{F}](x)$ denotes the discontinuity of a function $\mathcal{F}$ across $S$, and $q_{i}:=\partial_{i} \Phi$ is the wave covector. Given the constitutive law $H(F)$, which determines the excitation in terms of the field strength, the corresponding tensors $f_{i j}$ and $h_{i j}$, describing the jumps of the derivatives of field strength and excitation, are related by [22]

$$
h_{i j}=\frac{1}{2} \kappa_{i j}{ }^{k l} f_{k l}, \quad \text { with } \quad \kappa_{i j}{ }^{k l}:=\frac{\partial H_{i j}}{\partial F_{k l}} .
$$

We will call $\kappa_{i j}{ }^{k l}$ the jump tensor. In linear electrodynamics, its components coincide with the components of the constitutive tensor (which describes the linear law $H_{i j}$ $\left.=\frac{1}{2} \kappa_{i j}{ }^{k l} F_{k l}\right)$, and they are independent of the electromagnetic field. However, in general the jump tensor $\kappa_{i j}{ }^{k l}$ is a function of the electromagnetic field, the velocity of matter, the temperature, and other physical and geometrical variables. Quite remarkably, all the earlier results obtained for linear electrodynamics remain also valid in the general case because whatever local relation $H(F)$ may exist, the relation between the jumps of the field derivatives, according to Eq. (2.5), is always linear.

If we use Maxwell's equations (2.1), then Eqs. (2.3) and (2.4) yield

$$
\epsilon^{i j k l} q_{j} h_{k l}=0, \quad \epsilon^{i j k l} q_{j} f_{k l}=0 .
$$

Here $\epsilon^{i j k l}$ is the Levi-Civita tensor density of weight +1 , with $\epsilon^{0123}=1$. We will also use the Levi-Civita tensor density of weight -1 , which we denote by $\hat{\epsilon}_{i j k l}$, with $\hat{\epsilon}_{0123}$ $=1$.

Let us introduce the analogue of the conventional constitutive matrix

$$
\chi^{i j k l}:=\frac{1}{2} \epsilon^{i j m n} \kappa_{m n}{ }^{k l}=\frac{\partial \mathcal{H}^{i j}}{\partial F_{k l}},
$$

where we denote $\mathcal{H}^{i j}:=\frac{1}{2} \epsilon^{i j m n} H_{m n}$. Similarly to $\kappa_{i j}{ }^{k l}$, we will often call the tensor $\chi^{i j k l}$ the jump tensor density.

Now, making use of Eqs. (2.5) and (2.7), we rewrite the system (2.6) as

$$
\chi^{i j k l} q_{j} f_{k l}=0, \quad \epsilon^{i j k l} q_{j} f_{k l}=0 .
$$

Solving the last equation by $f_{i j}=q_{i} a_{j}-q_{j} a_{i}$, we finally reduce the first part of Eq. (2.8) to

$$
\chi^{i j k l} q_{j} q_{k} a_{l}=0 .
$$

This algebraic system has a nontrivial solution for $a_{i}$ only when the wave covectors satisfy a certain condition. The latter gives rise to our covariant Fresnel equation [20,21]

$$
\mathcal{G}^{i j k l}(\chi) q_{i} q_{j} q_{k} q_{l}=0,
$$

with the fourth order Fresnel tensor density $\mathcal{G}$ of weight +1 defined by

$$
\mathcal{G}^{i j k l}(\chi):=\frac{1}{4 !} \hat{\epsilon}_{m n p q} \hat{\epsilon}_{r s t u} \chi^{m n r(i} \chi^{j|p s| k} \chi^{l) q t u} .
$$

It is totally symmetric, $\mathcal{G}^{i j k l}(\chi)=\mathcal{G}^{(i j k l)}(\chi)$, and thus has 35 independent components. A particular case of that construction has been introduced by Tamm [23].

\section{LOCAL NONLINEAR ELECTRODYNAMICS}

Let us denote the two independent electromagnetic invariants as

$$
I_{1}:=F_{i j} F^{i j}, \quad I_{2}:=F_{i j} \widetilde{F}^{i j},
$$

where $\widetilde{F}^{i j}:=\frac{1}{2} \eta^{i j k l} F_{k l}$ and $\eta^{i j k l}:=(-g)^{-1 / 2} \epsilon^{i j k l}$. The Hodge operator for the exterior forms is denoted by as asterisk (*), as usual; and we use a tilde to denote the dual 2-tensors.

We will study the class of nonlinear electrodynamics models which are described by the Lagrangian 4-form

$$
V=L \eta, \quad \text { with } \quad L=L\left(I_{1}, I_{2}\right) .
$$


Here, as usual, $\eta$ is the 4-form of the spacetime volume. Our analysis is generally covariant, i.e., we do not restrict ourselves to Cartesian coordinates for which $g_{i j}=\operatorname{diag}(1,-1$, $-1,-1)$. We instead consider $g_{i j}$ as an arbitrary Lorentzian spacetime metric, although we do not take into account possible nonminimal coupling of the electromagnetic field to the spacetime curvature. In other words, we exclude the Lagrangian models with the curvature terms arising from the quantum corrections on a curved spacetime which were studied by Drummond and Hathrell [18], e.g. As a matter of fact, such terms can be straightforwardly included in our general approach using the general quartic Fresnel equation (2.10), (2.11) but this goes beyond the scope of the current paper. The corresponding generalization will be discussed elsewhere.

The electromagnetic excitation 2-form, which enters the Maxwell equation (2.1), is derived as the derivative of the Lagrangian form, $H=-\partial V / \partial F$. Explicitly, we then have the nonlinear constitutive law

$$
H=4\left(-L_{1}^{*} F+L_{2} F\right)
$$

We denote the partial derivatives of the Lagrangian function with respect to its arguments as

$$
L_{a}:=\frac{\partial L}{\partial I_{a}}, \quad L_{a b}:=\frac{\partial^{2} L}{\partial I_{a} \partial I_{b}}, \quad a, b=1,2 .
$$

In accordance with Eqs. (2.5) and (2.7), the direct differentiation of Eq. (3.3) yields the jump tensor density

$$
\begin{aligned}
\chi^{i j k l}= & \sqrt{-g}\left[k_{1} g^{i[k} g^{l] j}+k_{2} F^{i j} F^{k l}+k_{3} \widetilde{F}^{i j} F^{k l}\right. \\
& \left.+k_{4} F^{i j} \widetilde{F}^{k l}+k_{5} \widetilde{F}^{i j} \widetilde{F}^{k l}+k_{6} \eta^{i j k l}\right] .
\end{aligned}
$$

The coefficients $k_{A}, A=1, \ldots, 6$, are functions of the electromagnetic fields:

$$
\begin{gathered}
k_{1}=4 L_{1}, \quad k_{2}=8 L_{11}, \quad k_{3}=k_{4}=8 L_{12}, \\
k_{5}=8 L_{22}, \quad k_{6}=2 L_{2} .
\end{gathered}
$$

The identifications (3.6) are derived for the nonlinear Lagrangian (3.2) from the constitutive law (3.3). However, in most computations below we will consider the most general case with unspecified arbitrary coefficients $k_{A}$. This may be useful if we want to study nonlinear electrodynamics of a more general type, for instance, with dissipation effects and/or in moving media.

In general, the untwisted tensor density $\chi^{i j k l}(x)$ of weight +1 has 36 independent components. We can decompose it into irreducible pieces [21] with respect to the 6-dimensional ("bivector") linear group as follows:

$$
\chi^{i j k l}={ }^{(1)} \chi^{i j k l}+{ }^{(2)} \chi^{i j k l}+{ }^{(3)} \chi^{i j k l} .
$$

The irreducible pieces of $\chi$ are defined by

$$
\begin{aligned}
& { }^{(2)} \chi^{i j k l}:=\frac{1}{2}\left(\chi^{i j k l}-\chi^{k l i j}\right)=-{ }^{(2)} \chi^{k l i j}, \\
& { }^{(3)} \chi^{i j k l}:=\chi^{[i j k l]}, \\
& { }^{(1)} \chi^{i j k l}:=\chi^{i j k l-\left({ }^{(2)} \chi^{i j k l}-{ }^{(3)} \chi^{i j k l}={ }^{(1)} \chi^{k l i j} .\right.}
\end{aligned}
$$

The irreducible pieces ${ }^{(1)} \chi,{ }^{(2)} \chi$, and ${ }^{(3)} \chi$ have 20,15 , and 1 independent components, respectively. The possible presence of an axion piece ${ }^{(3)} \chi$ was first studied by $\mathrm{Ni}$ [24], whereas a constitutive law with an isotropic skewon ${ }^{(2)} \chi$ was discussed by Nieves and $\mathrm{Pal}$ [25].

In the Lagrangian models (3.2), the effective constitutive tensor is automatically symmetric, i.e., ${ }^{(2)} \chi=0$, which follows from Eq. (3.6), since $k_{3}=k_{4}$. However, in general the jump tensor density (3.5) has all three irreducible pieces:

$$
\begin{aligned}
{ }^{(1)} \chi^{i j k l}= & \sqrt{-g}\left[k_{1} g^{i[k} g^{l] j}+k_{2} F^{i j} F^{k l}+\frac{\left(k_{3}+k_{4}\right)}{2}\right. \\
& \times\left(\widetilde{F}^{i j} F^{k l}+F^{i j} \widetilde{F}^{k l}\right)+k_{5} \widetilde{F}^{i j} \widetilde{F}^{k l}-\frac{1}{12}\left[\left(k_{3}+k_{4}\right) I_{1}\right. \\
& \left.\left.+\left(k_{5}-k_{2}\right) I_{2}\right] \eta^{i j k l}\right] \\
{ }^{(2)} \chi^{i j k l}= & \frac{\left(k_{3}-k_{4}\right)}{2} \sqrt{-g}\left(\widetilde{F}^{i j} F^{k l}-F^{i j} \widetilde{F}^{k l}\right) \\
{ }^{(3)} \chi^{i j k l}= & \frac{1}{12} \sqrt{-g}\left[\left(k_{3}+k_{4}\right) I_{1}+\left(k_{5}-k_{2}\right) I_{2}+12 k_{6}\right] \eta^{i j k l} .
\end{aligned}
$$

\section{FRESNEL EQUATION AND BIREFRINGENCE}

Our analysis in the framework of the Hadamard formalism yields the Fresnel equation in the generally covariant form (2.10) with the Fresnel tensor density (2.11). For the explicit jump tensor density (3.5), it thus remains to substitute its components into Eq. (2.11). A straightforward calculation yields the result:

$$
\mathcal{G}^{i j k l}=-\frac{k_{1}}{8} \sqrt{-g}\left(\mathcal{X}^{(i j} g^{k l)}+2 \mathcal{Y} g^{(i j} t^{k l)}+\mathcal{Z} t^{(i j} t^{k l)}\right) .
$$

Here we denote

$$
\begin{aligned}
& \mathcal{X}=k_{1}^{2}+\frac{k_{1}}{2}\left(k_{3}+k_{4}\right) I_{2}-k_{1} k_{5} I_{1}+\frac{1}{4}\left(k_{3} k_{4}-k_{2} k_{5}\right) I_{2}^{2}, \\
& \mathcal{Y}=k_{1}\left(k_{2}+k_{5}\right)+\left(k_{3} k_{4}-k_{2} k_{5}\right) I_{1} \\
& \mathcal{Z}=4\left(k_{2} k_{5}-k_{3} k_{4}\right)
\end{aligned}
$$

and

$$
t^{i j}:=F^{i k} F_{k}^{j} .
$$


The latter is closely related to the Minkowski energymomentum tensor

$$
T^{i j}:=-t^{i j}+\frac{1}{4} I_{1} g^{i j}
$$

The most remarkable property of Eq. (4.1) is that it is obviously factorizable into a product of two second order tensors. Correspondingly, the quartic Fresnel surface of the wave normals reduces to the product of two second order surfaces:

$$
\begin{aligned}
\mathcal{G}^{i j k l}(\chi) q_{i} q_{j} q_{k} q_{l} & =\frac{-k_{1}}{8 \mathcal{X}}\left(g_{1}^{i j} q_{i} q_{j}\right)\left(g_{2}^{k l} q_{k} q_{l}\right) \\
& =\frac{-k_{1}}{8 \mathcal{Z}}\left(\bar{g}_{1}^{i j} q_{i} q_{j}\right)\left(\bar{g}_{2}^{k l} q_{k} q_{l}\right) .
\end{aligned}
$$

In other words, the wave normals lie not on the quartic surface but on one of the two cones which are determined by the pair of optical metric tensors:

$$
\begin{aligned}
& g_{1}^{i j}:=\mathcal{X} g^{i j}+\left(\mathcal{Y}+\sqrt{\mathcal{Y}^{2}-\mathcal{X Z}}\right) t^{i j}, \\
& g_{2}^{i j}:=\mathcal{X} g^{i j}+\left(\mathcal{Y}-\sqrt{\mathcal{Y}^{2}-\mathcal{X Z}}\right) t^{i j} .
\end{aligned}
$$

The second equality in Eq. (4.7) offers a different description of the cones by means of the conformally equivalent metric tensors:

$$
\begin{aligned}
& \bar{g}_{1}^{i j}:=\left(\mathcal{Y}-\sqrt{\mathcal{Y}^{2}-\mathcal{X Z}}\right) g^{i j}+\mathcal{Z} t^{i j}=\frac{1}{\mathcal{X}}\left(\mathcal{Y}-\sqrt{\mathcal{Y}^{2}-\mathcal{X Z}}\right) g_{1}^{i j}, \\
& \bar{g}_{2}^{i j}:=\left(\mathcal{Y}+\sqrt{\mathcal{Y}^{2}-\mathcal{X Z}}\right) g^{i j}+\mathcal{Z} t^{i j}=\frac{1}{\mathcal{X}}\left(\mathcal{Y}+\sqrt{\mathcal{Y}^{2}-\mathcal{X Z}}\right) g_{2}^{i j} .
\end{aligned}
$$

Thus, the general Fresnel analysis demonstrates that in any local nonlinear electrodynamics model (3.2) the quartic wave surface always reduces to two light cones. This is the birefringence effect which is thus a general feature of the local nonlinear models (3.2).

\section{PROPERTIES OF OPTICAL METRICS}

Let us discuss the results obtained in the previous section. The following general observations are in order.

The Fresnel equation is trivially satisfied for all wave covectors when $k_{1}=0$, see Eq. (4.1). Thus, in order to have waves, every electrodynamical Lagrangian $L$ should necessarily depend on the invariant $I_{1}=F_{i j} F^{i j}$ (thus providing $\left.k_{1} \neq 0\right)$. Accordingly, we will always assume that $k_{1} \neq 0$.

In order to have a decent light propagation, the optical metrics should be real and with Lorentzian signature. How can one be a priori sure that for every $L$ an optical metric necessarily has these properties?

Using Eqs. (4.2)-(4.4) we find an explicit expression for the quantity under the square root in the above formulas:

$$
\mathcal{Y}^{2}-\mathcal{X Z}=N_{1}^{2}+N_{2} N_{3},
$$

where we have denoted

$$
\begin{aligned}
& N_{1}:=k_{1}\left(k_{2}-k_{5}\right)+\left(k_{3} k_{4}-k_{2} k_{5}\right) I_{1}, \\
& N_{2}:=2 k_{1} k_{3}+\left(k_{3} k_{4}-k_{2} k_{5}\right) I_{2}, \\
& N_{3}:=2 k_{1} k_{4}+\left(k_{3} k_{4}-k_{2} k_{5}\right) I_{2} .
\end{aligned}
$$

The expression (5.1) is always non-negative in every nonlinear theory (3.2) because $N_{2}=N_{3}$ when we take into account that $k_{3}=k_{4}$, see Eq. (3.6).

The signature of a four-dimensional metric is Lorentzian if and only if its determinant is negative. Straightforward computation yields, for the optical metrics (4.8), (4.9);

$$
\begin{aligned}
\left(\operatorname{det}_{a}^{i j}\right)=\left(\operatorname{det} g^{i j}\right) & {\left[\alpha^{2}+\frac{\alpha \beta_{a}}{2} I_{1}-\frac{\beta_{a}^{2}}{16} I_{2}^{2}\right]^{2}, } \\
a & =1,2 .
\end{aligned}
$$

Here $\alpha=\mathcal{X}$ and $\beta_{1}=\mathcal{Y}+\sqrt{\mathcal{Y}^{2}-\mathcal{X Z}}, \beta_{2}=\mathcal{Y}-\sqrt{\mathcal{Y}^{2}-\mathcal{X Z}}$. As we see, both optical metrics have Lorentzian signature as soon as the spacetime metric $g^{i j}$ is Lorentzian.

Summarizing, we have demonstrated that Eqs. (4.8) and (4.9) indeed describe the generic effect of a birefringent light propagation for all local nonlinear Lagrangians (3.2).

Recently, the emergence of the two "effective geometries" has been described in $[14,15]$ without using the Fresnel approach. These results agree with those obtained in our analysis, since one can show that our optical metrics are conformally equivalent to the effective metrics of $[14,15]$. Moreover, one can recast the optical metrics (4.8)-(4.11) into the form of the so-called Boillat metrics of [16].

\section{SPECIAL LAGRANGIANS}

It is worthwhile to discuss certain particular nonlinear models which are potentially of physical interest.

\section{A. Lagrangian $L=L\left(I_{2}\right)$}

When the Lagrangian depends only on the second electromagnetic invariant, we have $k_{1}=0$, and there are no waves in such models.

\section{B. Lagrangian $L=L\left(I_{1}\right)$}

For the Lagrangian which, on the contrary, depends on the first invariant only, we find $k_{3}=k_{4}=k_{5}=0$. Accordingly, from Eqs. (4.2) $-(4.4)$ we find $\mathcal{X}=k_{1}^{2}, \mathcal{Y}=k_{1} k_{2}$, and $\mathcal{Z}=0$, and thus Eqs. (4.8) and (4.9) yield

$$
g_{1}^{i j}=k_{1}\left(k_{1} g^{i j}+2 k_{2} t^{i j}\right), \quad g_{2}^{i j}=k_{1}^{2} g^{i j} .
$$

Correspondingly, we still have birefringence with some photons moving along the standard null rays of the spacetime metric $g^{i j}$, whereas other photons choosing the rays null with respect to the optical metric $L_{1} g^{i j}+4 L_{11} t^{i j}$, cf. [14]. 


\section{Lagrangian $L=U\left(I_{1}\right)+\alpha I_{2}$}

This is a simple generalization of the previous case. Here $\alpha$ does not depend on the electromagnetic field, although it is not a constant, in general. When it depends on the spacetime coordinates, $\alpha=\alpha(x)$, one can identify it with the axion field, cf. [24].

Here we again have $k_{3}=k_{4}=k_{5}=0$ and we recover the same light cone structure (6.1). To put it differently, the axion field does not disturb the light cones which are solely determined by the spacetime metric and by the dependence of the Lagrangian on the invariant $I_{1}$.

\section{Lagrangian $L=a I_{1}+V\left(I_{2}\right)$}

Then $k_{1}=4 a, k_{2}=k_{3}=k_{4}=0$, and $k_{5}=8 V^{\prime \prime}$, which yields $\mathcal{X}=16 a^{2}-32 V^{\prime \prime} I_{1}, \mathcal{Y}=32 a V^{\prime \prime}$, and $\mathcal{Z}=0$. Consequently,

$$
\begin{aligned}
& g_{1}^{i j}=16 a\left[\left(a-2 V^{\prime \prime} I_{1}\right) g^{i j}+4 V^{\prime \prime} t^{i j}\right], \\
& g_{2}^{i j}=16 a\left(a-2 V^{\prime \prime} I_{1}\right) g^{i j},
\end{aligned}
$$

i.e., there is again birefringence with one cone determined by the standard spacetime metric.

\section{E. Heisenberg-Euler Lagrangian}

In the one-loop approximation, polarization of vacuum in quantum electrodynamics is described by the HeisenbergEuler effective Lagrangian [2-4]. The critical magnetic field $B_{k}=\left(m^{2} c^{2} / e \hbar\right) \approx 4.4 \times 10^{9} \mathrm{~T}$ (for the electron mass $m$ and charge $e$ ) essentially determines the physical structure of the nonlinear model. For the weak fields which satisfy $I_{1}$ $\ll B_{k}^{2}, I_{2} \ll B_{k}^{2}$ the Heisenberg-Euler Lagrangian reads

$L=-\frac{1}{4} I_{1}+\frac{\alpha}{90 B_{k}^{2}}\left[I_{1}^{2}+\frac{7}{4} I_{2}^{2}-\frac{I_{1}}{B_{k}^{2}}\left(\frac{2}{7} I_{1}^{2}+\frac{13}{28} I_{2}^{2}\right)\right]$.

Here $\alpha=\left(e^{2} / 4 \pi \hbar c\right)$ is the fine structure constant. From this Lagrangian we find, keeping only the leading order terms,

$$
\begin{aligned}
& \mathcal{X}=1+\frac{6 \alpha I_{1}}{45 B_{k}^{2}}, \\
& \mathcal{Y}=\frac{\alpha}{45 B_{k}^{2}}\left(-22+\frac{74}{7} \frac{I_{1}}{B_{k}^{2}}\right), \\
& \mathcal{Z}=\left(\frac{\alpha}{45 B_{k}^{2}}\right)^{2}\left(448-\frac{3520}{7} \frac{I_{1}}{B_{k}^{2}}\right) .
\end{aligned}
$$

As a result, we obtain the optical metrics

$$
\begin{aligned}
& g_{1}^{i j}=\left(1+\frac{6 \alpha I_{1}}{45 B_{k}^{2}}\right) g^{i j}-\frac{16 \alpha}{45 B_{k}^{2}} t^{i j}, \\
& g_{2}^{i j}=\left(1+\frac{6 \alpha I_{1}}{45 B_{k}^{2}}\right) g^{i j}-\frac{28 \alpha}{45 B_{k}^{2}} t^{i j} .
\end{aligned}
$$

Thus we have birefringence and both light cones differ from the one determined by the spacetime metric, cf. also [14].

\section{F. Born-Infeld theory}

The Lagrangian of the Born-Infeld (BI) theory [1] reads

$$
L=b^{2}\left(1-\sqrt{1+\frac{1}{2 b^{2}} I_{1}-\frac{1}{16 b^{4}} I_{2}^{2}}\right) .
$$

Here $b$ is the coupling constant which has the meaning of the critical field. Correspondingly,

$$
\begin{aligned}
& \mathcal{X}=\frac{\left(1+\frac{1}{2 b^{2}} I_{1}\right)^{2}}{\left(1+\frac{1}{2 b^{2}} I_{1}-\frac{1}{16 b^{4}} I_{2}^{2}\right)^{2}}, \\
& \mathcal{Y}=\frac{-\left(1+\frac{1}{2 b^{2}} I_{1}\right)}{b^{2}\left(1+\frac{1}{2 b^{2}} I_{1}-\frac{1}{16 b^{4}} I_{2}^{2}\right)^{2}}, \\
& \mathcal{Z}=\frac{1}{b^{4}\left(1+\frac{1}{2 b^{2}} I_{1}-\frac{1}{16 b^{4}} I_{2}^{2}\right)^{2}} .
\end{aligned}
$$

As a result, we find $\mathcal{Y}^{2}-\mathcal{X Z}=0$, and thus the two optical metrics (4.8) and (4.9) coincide,

$$
\begin{aligned}
g_{1}^{i j}= & g_{2}^{i j}=\frac{\left(1+\frac{1}{2 b^{2}} I_{1}\right)}{\left(1+\frac{1}{2 b^{2}} I_{1}-\frac{1}{16 b^{4}} I_{2}^{2}\right)^{2}} \\
& \times\left[\left(1+\frac{1}{2 b^{2}} I_{1}\right) g^{i j}-\frac{1}{b^{2}} t^{i j}\right] .
\end{aligned}
$$

Birefringence disappears, and the photons propagate along a single light cone determined by the "quasi-metric" of Plebanski [11]

$$
\left(1+\frac{1}{2 b^{2}} I_{1}\right) g^{i j}-\frac{1}{b^{2}} t^{i j}
$$

\section{VELOCITY OF LIGHT}

The knowledge of the optical metric is sufficient for determining the velocity of light. The phase velocity $v$ of wave propagation is defined by the wave covector components by means of the relation

$$
q_{a}=\frac{q_{0}}{v} k_{a}, \quad a=1,2,3
$$


where $k_{a}$ are the components of the unit 3-covector. Although we confine our attention to the phase velocity, it is straightforward to show that for the models under consideration the group velocity is always greater or equal to the phase velocity. Substituting Eq. (7.1) into the light cone equations $g_{1}^{i j} q_{i} q_{j}=0$ and $g_{2}^{i j} q_{i} q_{j}=0$, one can find the velocities of light explicitly. Usually, of interest is the mean velocity value which is obtained after averaging over the directions of propagation and polarizations. A direct computation using the general form of the optical metrics (4.8) and (4.9) yields, in Cartesian coordinates,

$$
\begin{aligned}
\left\langle v^{2}\right\rangle= & \left(1+\frac{4}{3} T^{00} \frac{\mathcal{Y}+\mathcal{Z} t^{00}}{\mathcal{X}+2 \mathcal{Y} t^{00}+\mathcal{Z}\left(t^{00}\right)^{2}}\right) \\
& +\frac{2}{3} \delta_{a b} t^{0 a} t^{0 b} \frac{2 \mathcal{Y}^{2}-\mathcal{X Z}+\mathcal{Z}\left(t^{00}\right)^{2}+2 \mathcal{Y Z} t^{00}}{\left[\mathcal{X}+2 \mathcal{Y} t^{00}+\mathcal{Z}\left(t^{00}\right)^{2}\right]^{2}}
\end{aligned}
$$

In accordance with Eq. (4.6), $T^{00}=-t^{00}+I_{1} / 4=\left(\mathbf{B}^{2}\right.$ $\left.+\mathbf{E}^{2}\right) / 2$ is the energy density of the Maxwell field, whereas $\delta_{a b} t^{0 a} t^{0 b}=\mathbf{S}^{2}$ where $\mathbf{S}=\mathbf{E} \times \mathbf{B}$ is the energy flux density.

\section{A. Velocity shift in a polarized vacuum}

As an application of the general formula (7.2), let us obtain the velocity of light in a polarized vacuum. For the weak field limit of the Heisenberg-Euler electrodynamics, substituting Eqs. (6.4)-(6.6) into Eq. (7.2), we find, in the leading order,

$$
\left\langle v^{2}\right\rangle=1-\frac{88 \alpha}{135}\left(\frac{\mathbf{B}^{2}+\mathbf{E}^{2}}{2 B_{k}^{2}}\right) .
$$

This value of the mean velocity shift is in agreement with the previous results derived by different methods $[13,19]$.

\section{B. Light velocity in the Born-Infeld theory}

Although there is no birefringence in the Born-Infeld model, the velocity of light in it is different from $c^{2}=1$. We can use the same general formula (7.2) to find its value. Using Eqs. (6.10)-(6.12), we obtain

$$
\left\langle v^{2}\right\rangle=1-\frac{2}{3} \frac{\mathbf{E}^{2}+\mathbf{B}^{2}}{b^{2}+\mathbf{B}^{2}}+\frac{2}{3} \frac{\mathbf{E}^{2} \mathbf{B}^{2}-(\mathbf{E} \cdot \mathbf{B})^{2}}{\left(b^{2}+\mathbf{B}^{2}\right)^{2}} .
$$

It is worthwhile to note that in contrast to the approximate formula (7.3) which is valid only for the weak fields, the velocity shift (7.4) is the exact relation which is applicable to arbitrarily strong electromagnetic fields. Since evidently $\left(\mathbf{E}^{2}+\mathbf{B}^{2}\right)\left(b^{2}+\mathbf{B}^{2}\right)+(\mathbf{E} \cdot \mathbf{B})^{2}-\mathbf{E}^{2} \mathbf{B}^{2} \geqslant 0$, the velocity $\left\langle v^{2}\right\rangle$ is always less than or equal to 1 . It is remarkable though that due to the existence of the critical (maximal possible) value of the field $b$, the averaged velocity of light in the BornInfeld model cannot be arbitrarily small. It follows from Eq. (7.4) that the minimum possible value of $\left\langle v^{2}\right\rangle$ is $1 / 3$ for $\mathbf{E}^{2}$ $=b^{2}$ and $\mathbf{B}$ zero or (anti-)parallel to $\mathbf{E}$.

\section{NO BIREFRINGENCE CONDITION}

In this section, we will restrict our attention to the Lagrangian theories for which the constitutive tensor is Eq. (3.5), and the coefficients are derived as Eq. (3.6). It is important that the Fresnel analysis reveals that $k_{1} \neq 0$, otherwise there is no decent wave propagation at all.

As it is clear from Eq. (5.1), the necessary and sufficient condition of the absence of birefringence is provided by the pair of equations:

$$
\begin{aligned}
& N_{1}=k_{1}\left(k_{2}-k_{5}\right)+\left(k_{3} k_{4}-k_{2} k_{5}\right) I_{1}=0, \\
& N_{2}=N_{3}=2 k_{1} k_{3}+\left(k_{3} k_{4}-k_{2} k_{5}\right) I_{2}=0 .
\end{aligned}
$$

Here the property $k_{3}=k_{4}$ of the Lagrangian models is used.

Taking into account that all $k$ 's are the partial derivatives of the Lagrangian $L$ with respect to $I_{1}$ and/or $I_{2}$ as displayed in Eq. (3.6), we can view the above system as a pair of partial differential equations, the solution $L=L\left(I_{1}, I_{2}\right)$ of which describes a model without birefringence (i.e., with a single light cone). At least two such particular solutions are already known: one is rather simple, namely, the standard Maxwell theory with $L=-I_{1} / 4$. Another is more nontrivial, this is the Born-Infeld theory with the Lagrangian (6.9). One may ask the question: Are these the only solutions of the system (8.1), (8.2)? The immediate inspection of the system (8.1), (8.2) shows that the answer is negative. For example, the Lagrangian function $L\left(I_{1}, I_{2}\right)=a I_{1} / I_{2}$, with constant $a$, satisfies the equations (8.1), (8.2). Such a nonlinear (and nonpolynomial) model thus also has no birefringence. It is an open problem to find the complete set of solutions of Eqs. (8.1), (8.2), leading then to a single light cone.

\section{CLOSURE CONDITION}

Let us denote the "traceless" part of the jump tensor density as

$$
\mathcal{K}^{i j k l}:={ }^{(1)} \chi^{i j k l}+{ }^{(2)} \chi^{i j k l} .
$$

As we know [21], only the traceless part determines the Fresnel surface, whereas the axion part ${ }^{(3)} \chi^{i j k l}$ drops out completely from the wave propagation analysis.

For the general jump tensor density (3.5), we find, after some algebra,

$$
\begin{aligned}
\frac{1}{4} \hat{\epsilon}_{p q i j} \mathcal{K}^{i j k l} \hat{\epsilon}_{k l r s} \mathcal{K}^{r s m n}= & \left(-k_{1}^{2}+\frac{a_{0}^{2}}{k_{1}^{2}}\right) \delta_{p}^{[m} \delta_{q}^{n]} \\
& +a_{0} \eta_{p q}{ }^{m n}+a_{1} F_{p q} \widetilde{F}^{m n}+a_{2} \widetilde{F}_{p q} F^{m n} \\
& +a_{3} F_{p q} F^{m n}+a_{4} \widetilde{F}_{p q} \widetilde{F}^{m n} .
\end{aligned}
$$

Here the coefficients read: 


$$
\begin{aligned}
& a_{0}=\frac{k_{1}}{6}\left[\left(k_{3}+k_{4}\right) I_{1}+\left(k_{5}-k_{2}\right) I_{2}\right] \\
& =\frac{1}{6}\left[-N_{1} I_{2}+\frac{\left(N_{2}+N_{3}\right)}{2} I_{1}\right] \text {, } \\
& a_{1}=-\left(k_{3}+k_{4}\right) k_{1}-k_{3} k_{4} I_{2} \\
& +\frac{k_{5}}{3}\left[2\left(k_{3}+k_{4}\right) I_{1}+\left(2 k_{5}+k_{2}\right) I_{2}\right] \\
& =N_{1}\left(-\frac{2 k_{5}}{3 k_{1}} I_{2}\right)+\frac{\left(N_{2}+N_{3}\right)}{2}\left(-1+\frac{2 k_{5}}{3 k_{1}} I_{1}\right) \text {, } \\
& a_{2}=-\left(k_{3}+k_{4}\right) k_{1}-k_{3} k_{4} I_{2} \\
& +\frac{k_{2}}{3}\left[2\left(k_{3}+k_{4}\right) I_{1}+\left(k_{5}+2 k_{2}\right) I_{2}\right] \\
& =N_{1}\left(\frac{2 k_{2}}{3 k_{1}} I_{2}\right)+\frac{\left(N_{2}+N_{3}\right)}{2}\left(-1-\frac{2 k_{2}}{3 k_{1}} I_{1}\right) \text {, } \\
& a_{3}=\left(k_{5}-k_{2}\right) k_{1}+k_{2} k_{5} I_{1} \\
& +\frac{k_{3}}{3}\left[2\left(k_{5}-k_{2}\right) I_{2}+\left(2 k_{3}-k_{4}\right) I_{1}\right] \\
& =N_{1}\left(-1-\frac{2 k_{3}}{3 k_{1}} I_{2}\right)+\frac{\left(N_{2}+N_{3}\right)}{2}\left(\frac{2 k_{3}}{3 k_{1}} I_{1}\right), \\
& a_{4}=-\left(k_{5}-k_{2}\right) k_{1}-k_{2} k_{5} I_{1} \\
& -\frac{k_{4}}{3}\left[2\left(k_{5}-k_{2}\right) I_{2}+\left(2 k_{4}-k_{3}\right) I_{1}\right] \\
& =N_{1}\left(1+\frac{2 k_{4}}{3 k_{1}} I_{2}\right)+\frac{\left(N_{2}+N_{3}\right)}{2}\left(-\frac{2 k_{4}}{3 k_{1}} I_{1}\right) \text {. }
\end{aligned}
$$

The third lines in Eqs. (9.4)-(9.7) give the $a$ 's in terms of the combinations (8.1)-(8.2). Certainly, we use the assumption that $k_{1} \neq 0$.

Like the constitutive tensor of linear electrodynamics, the jump tensor $\kappa_{i j}{ }^{k l}:=\frac{1}{2} \hat{\epsilon}_{i j m n} \chi^{m n k l}$ determines a linear map in the six-dimensional space of 2-forms. When the action of this map, repeated twice, brings us (up to a factor) back to the identity map, we speak of the closure property of $\kappa_{i j}{ }^{k l}$. The importance of the closure property is related to the fact that ultimately $\kappa_{i j}{ }^{k l}$ turns out to be a duality operator which determines a unique conformal Lorentzian metric on the spacetime.

In local nonlinear electrodynamics, the jump tensor density (3.5) has the closure property when

$$
a_{0}=0, \quad a_{1}=0, \quad a_{2}=0, \quad a_{3}=0, \quad a_{4}=0,
$$

as is evident from Eq. (9.2).

\section{EQUIVALENCE OF CLOSURE AND NO BIREFRINGENCE CONDITIONS}

In linear electrodynamics, there is much evidence (although the final rigorous proof is still missing) that the quartic Fresnel surface of wave covectors reduces to a unique light cone if and only if the constitutive tensor has the closure property.

For the local nonlinear Lagrangian theories (3.2) it is possible to make some progress in solving the equivalence problem. In a certain sense, the situation here is simpler because we have discovered that the quartic Fresnel surface is always reduced to the product of the light cones (birefringence). The next step is thus to study under which conditions the birefringence disappears and, correspondingly, a unique light cone arises.

Theorem. For the nonlinear electrodynamical models described by the Lagrangian (3.2), the Fresnel equation implies a single light cone (no birefringence) if and only if the traceless part of the jump tensor density satisfies the closure property.

Proof. As a preliminary remark, we note that for the Lagrangian models (3.2) the jump tensor density (3.5) is symmetric because $k_{3}=k_{4}$. As a result, $N_{2}=N_{3}$.

The necessary condition is evident. The birefringence is absent when $N_{1}=N_{2}\left(=N_{3}\right)=0$, see Eq. (8.2). Then we immediately read from Eqs. (9.3)-(9.7) that $a_{0}=a_{1}=a_{2}=a_{3}$ $=a_{4}=0$, and thus the closure is recovered from Eq. (9.2).

The sufficient condition is also proved straightforwardly. The closure condition (9.8) has the unique solution

$$
N_{1}=0, \quad \frac{N_{2}+N_{3}}{2}=0,
$$

when we analyze Eqs. (9.3)-(9.7). Since for the Lagrangian models (3.2) we have $N_{2}=N_{3}$, then Eq. (10.1) yields $N_{1}$ $=N_{2}=N_{3}=0$. Thus, there is no birefringence.

To put it differently, we have proven that the closure of the traceless jump tensor density is the necessary and sufficient condition for the reduction of the fourth order Fresnel wave surface to a single light cone. This is true for all nonlinear Lagrangian electrodynamical theories (3.2). Returning to our studies of the general linear electrodynamics, we expect that a similar result holds true there.

\section{On asymmetric jump tensors}

Symmetry of the jump tensor density is very important in the equivalence proof above. In order to clarify this point, let us consider an arbitrary jump tensor density (3.5) without assuming the explicit form of the coefficients (3.6). When $k_{3} \neq k_{4}$, the jump tensor density has the nontrivial skewon part (3.11). Also, $N_{2} \neq N_{3}$ [in fact, as we can see from Eqs. (5.3) and (5.4), $\left.N_{2}-N_{3}=2 k_{1}\left(k_{3}-k_{4}\right)\right]$.

We can easily verify that the closure of an asymmetric operator is not equivalent to the no-birefringence property. Indeed, take, for instance, $k_{1} \neq 0, k_{2}=k_{3}=0, k_{4} \neq 0$, and $k_{5}=0$. Then the jump tensor density is asymmetric and $N_{1}$ $=N_{2}=0$, but $N_{3} \neq 0$. We then obtain a unique light cone because Eq. (5.1) vanishes identically. However, the closure 
condition (9.8) is not satisfied, since Eqs. (9.3)-(9.7) are nontrivial for $N_{1}=N_{2}=0$ and $N_{3} \neq 0$. This also means that the requirement of a unique light cone does not necessarily imply that $\chi$ must be symmetric.

The opposite is also true: Suppose an asymmetric jump tensor density (3.5) has the closure property, i.e., Eq. (9.8) is fulfilled. Then we find Eq. (10.1) again. However, the second part of Eq. (10.1) yields $N_{2}=-N_{3}$, and consequently Eq. (5.1) together with Eqs. (4.8) and (4.9) describe the case of a birefringent and dissipative wave propagation.

These examples show that the closure of an asymmetric jump (or constitutive) tensor density does not guarantee the absence of birefringence, and, vice versa, no-birefringence is not accompanied by the closure property for an asymmetric operator.

\section{MOVING ISOTROPIC NONLINEAR MEDIA}

Recently, there has been some interest in the light propagating in moving media with nontrivial dielectric and magnetic properties. The first covariant analysis of the Fresnel equation for this case was done by Kremer [26]. An isotropic medium is characterized by the constitutive law

$$
\mathcal{H}^{i j}=\sqrt{-g}\left[\frac{1}{\mu} F^{i j}+2\left(\frac{1}{\mu}-\varepsilon\right) u_{k} F^{k[i} u^{j]}\right],
$$

where $u^{i}$ is the 4-velocity of the moving matter (normalized as usual by $u_{i} u^{i}=1$ ), and $\varepsilon$ and $\mu$ are the permeability and permittivity functions of the isotropic medium. The case when they do not depend on the electromagnetic field strength (being constant in space and time, for example) was investigated in [26].

More recently, the nonlinear case when $\varepsilon=\varepsilon(F)$ and $\mu$ $=\mu(F)$ are functions of the electromagnetic field has been studied by De Lorenci et al. [15]. However, the attention was restricted to certain special cases, and the general result is still missing.

We can perform a fairly complete analysis of the wave propagation in a nonlinear moving media on the basis of our covariant Fresnel equation (2.10). By differentiation, we easily find the jump tensor density (2.7):

$$
\begin{aligned}
\chi^{i j k l}= & \sqrt{-g}\left[\frac{2}{\mu} g^{i[k} g^{l] j}+4\left(\frac{1}{\mu}-\varepsilon\right) u^{[k} g^{l][i} u^{j]}\right. \\
& \left.+m^{k l} F^{i j}+2\left(m^{k l}-e^{k l}\right) u_{m} F^{m[i} u^{j]}\right] .
\end{aligned}
$$

The second line is absent in the linear theory, and the tensors

$$
m^{i j}:=\frac{\partial(1 / \mu)}{\partial F_{i j}}, \quad e^{i j}:=\frac{\partial \varepsilon}{\partial F_{i j}},
$$

are responsible for the nonlinear electrodynamical effects.

Inspection immediately reveals that the jump tensor density (11.2) contains both an axion and a skewon part. There are claims in the literature that axion and skewon, in general, do not have physical sense. However, here we encounter a simple and a physically sound example with both pieces being nontrivial. The irreducible pieces (3.8), (3.9) read:

$$
\begin{aligned}
{ }^{(1)} \chi^{i j k l}= & \sqrt{-g}\left[\frac{2}{\mu} g^{i[k} g^{l] j}+4\left(\frac{1}{\mu}-\varepsilon\right) u^{[k} g^{l][i} u^{j]}\right. \\
& +\frac{1}{2}\left(m^{i j} F^{k l}+m^{k l} F^{i j}\right)+\left(m^{k l}-e^{k l}\right) P^{[i} u^{j]} \\
& +\left(m^{i j}-e^{i j}\right) P^{[k} u^{l]} \\
& \left.+\frac{1}{12} \eta^{i j k l}\left[F^{m n} \tilde{m}_{m n}+2\left(\tilde{m}_{m n}-\tilde{e}_{m n}\right) P^{m} u^{n}\right]\right],
\end{aligned}
$$

$$
\begin{aligned}
{ }^{(2)} \chi^{i j k l}= & \sqrt{-g}\left[\frac{1}{2}\left(F^{i j} m^{k l}-F^{k l} m^{i j}\right)\right. \\
& \left.+\left(m^{k l}-e^{k l}\right) P^{[i} u^{j]}-\left(m^{i j}-e^{i j}\right) P^{[k} u^{l]}\right],
\end{aligned}
$$

and for the axion piece, we have

$\hat{\epsilon}_{i j k l} \chi^{i j k l}=\sqrt{-g} \hat{\epsilon}_{i j k l}\left[F^{i j} m^{k l}+2\left(m^{k l}-e^{i j}\right) P^{i} u^{j}\right]$.

Here we have denoted $P^{j}:=u_{i} F^{i j}$. Thus, nonlinear isotropic matter does have axion and skewon induced by nonlinearity.

\section{Nonmagnetic matter}

Let us consider the case when the magnetic constant is independent of the electromagnetic field, that is $m^{i j}=0$. [In the simplest case, we can restrict the attention to the purely dielectric medium with $\mu=1$. However, we will formally keep $\mu \neq 1$, for the sake of generality.]

It is convenient to make the evident split of Eq. (11.2) into the sum $\chi^{i j k l}=\phi^{i j k l}+\psi^{i j k l}$, with

$$
\begin{aligned}
\phi^{i j k l} & =\sqrt{-g}\left[\frac{2}{\mu} g^{i[k} g^{l] j}+4\left(\frac{1}{\mu}-\varepsilon\right) u^{[k} g^{l][i} u^{j]}\right], \\
\psi^{i j k l} & =-2 \sqrt{-g} e^{k l} u_{m} F^{m[i} u^{j]} .
\end{aligned}
$$

It is straightforward to find the Fresnel tensor for the first piece:

$$
\left.\mathcal{G}^{i j k l}(\phi)=-\frac{\varepsilon}{\mu^{2}} \sqrt{-g} g^{(i j} g^{\circ} k l\right)
$$

Here we have denoted the so-called Gordon optical metric $[27]$ as

$$
\stackrel{\circ}{g}^{i j}:=g^{i j}+(\varepsilon \mu-1) u^{i} u^{j} .
$$

Its inverse reads

$$
\stackrel{\circ}{g}_{i j}:=g_{i j}+\left(\frac{1}{\varepsilon \mu}-1\right) u_{i} u_{j}
$$


and the determinant can be easily computed: $\operatorname{det} \stackrel{\circ}{g}$ $=(\operatorname{det} g) /(\varepsilon \mu)$.

Using this optical metric we can simplify the form of Eq. (11.7), bringing it to

$$
\phi^{i j k l}=\frac{2}{\mu} \sqrt{-g} g^{i\left[k^{\circ} g\right] j}=2 \sqrt{\frac{\varepsilon}{\mu}} \sqrt{|\stackrel{\circ}{g}|{ }^{\circ}}{ }^{i\left[k^{\circ}\right.}{ }^{\circ] j} .
$$

Now, for $\chi=\phi+\psi$, using a compact notation by omitting the indices, we have

$$
\mathcal{G}(\chi)=\mathcal{G}(\phi)+\mathcal{G}(\psi)+\frac{1}{4 !}\left(O_{1}+O_{2}+O_{3}+T_{1}+T_{2}+T_{3}\right)
$$

Here the mixed terms $O_{a}$ contain one $\psi$ factor and the $T_{a}$ 's two $\psi$ factors. Postponing the symmetrization over $i, j, k, l$ to the very last moment, these terms read explicitly as follows:

$$
\begin{aligned}
& O_{1}^{i j k l}(\phi, \psi, \phi)=\hat{\epsilon}_{m n p q} \hat{\epsilon}_{r s t u} \phi^{m n r i} \psi^{j p s k} \phi^{l q t u}, \\
& O_{2}^{i j k l}(\psi, \phi, \phi)=\hat{\boldsymbol{\epsilon}}_{m n p q} \hat{\boldsymbol{\epsilon}}_{r s t u} \psi^{m n r i} \phi^{j p s k} \phi^{l q t u}, \\
& O_{3}^{i j k l}(\phi, \phi, \psi)=\hat{\boldsymbol{\epsilon}}_{m n p q} \hat{\boldsymbol{\epsilon}}_{r s t u} \phi^{m n r i} \phi^{j p s k} \psi^{l q t u}, \\
& T_{1}^{i j k l}(\psi, \phi, \psi)=\hat{\boldsymbol{\epsilon}}_{m n p q} \hat{\boldsymbol{\epsilon}}_{r s t u} \psi^{m n r i} \phi^{j p s k} \psi^{l q t u}, \\
& T_{2}^{i j k l}(\psi, \psi, \phi)=\hat{\boldsymbol{\epsilon}}_{m n p q} \hat{\boldsymbol{\epsilon}}_{r s t u} \psi^{m n r i} \psi^{j p s k} \phi^{l q t u}, \\
& T_{3}^{i j k l}(\phi, \psi, \psi)=\hat{\boldsymbol{\epsilon}}_{m n p q} \hat{\boldsymbol{\epsilon}}_{r s t u} \phi^{m n r i} \psi^{j p s k} \psi^{l q t u} .
\end{aligned}
$$

An important observation is that all $T$ 's are vanishing for Eq. (11.8). Indeed, since

$$
\psi^{i j k l}=-2 \sqrt{-g} P^{[i} u^{j]} e^{k l},
$$

we straightforwardly find, for example,

$$
\begin{aligned}
T_{1}^{i j k l}(\psi, \chi, \psi)= & -4 g \hat{\epsilon}_{m n p q} \hat{\epsilon}_{r s t u} P^{[m} u^{n]} e^{r i} P^{[l} u^{q]} e^{t u} \chi^{j p s k} \\
= & -2 g \hat{\epsilon}_{m n p q} \hat{\epsilon}_{r s t u} \chi^{j p s k} P^{m} u^{n} \\
& \times\left(P^{l} u^{q}-P^{q} u^{l}\right) e^{r i} e^{s k} \\
= & 0 .
\end{aligned}
$$

This is zero because either the symmetric $u^{n} u^{q}$ or symmetric $P^{m} P^{q}$ is contracted with the antisymmetric $\hat{\epsilon}_{m n p q}$. Note that we on purpose write $\chi^{j p s k}$ as the second argument, because its form is arbitrary, not necessarily equal to Eq. (11.7). Analogously, we find:

$$
\begin{aligned}
T_{2}^{i j k l}(\psi, \psi, \chi)= & -4 g \hat{\epsilon}_{m n p q} \hat{\epsilon}_{r s t u} P^{[m} u^{n]} e^{r i} P^{[j} u^{p]} e^{s k} \chi^{l q t u} \\
= & -2 g \hat{\epsilon}_{m n p q} \hat{\epsilon}_{r s t u} \chi^{l q t u} P^{m} u^{n} \\
& \times\left(P^{j} u^{p}-P^{p} u^{j}\right) e^{r i} e^{s k} \\
= & 0
\end{aligned}
$$

It is a little bit more nontrivial to prove that $T_{3}$ also vanishes. We have, explicitly:

$$
\begin{aligned}
T_{3}^{i j k l}(\chi, \psi, \psi)= & -4 g \hat{\epsilon}_{m n p q} \hat{\epsilon}_{r s t u} P^{[j} u^{p]} e^{s k} P^{[l} u^{q]} e^{t u} \chi^{m n r i} \\
= & -g \hat{\epsilon}_{m n p q} \hat{\epsilon}_{r s t u} \chi^{m n r i}\left(P^{j} u^{p} P^{l} u^{q}\right. \\
& \left.-P^{p} u^{j} P^{l} u^{q}-P^{j} u^{p} P^{q} u^{l}+P^{p} u^{j} P^{q} u^{l}\right) e^{s k} e^{t u} \\
= & 0 .
\end{aligned}
$$

The first and the last terms in the parentheses contain the symmetric combinations $u^{p} u^{q}$ and $P^{p} P^{q}$ which are vanishing when contracted with the antisymmetric $\hat{\epsilon}_{m n p q}$. The two remaining terms in the parentheses are reduced, by means of a relabeling of indices, to $-P^{p} u^{q}\left(P^{l} u^{j}-P^{j} u^{l}\right)$. Recalling that at the end we impose the symmetrization over the free indices $(i, j, k, l)$, we thus prove that $T_{3}(\chi, \psi, \psi)=0$.

Since in all the three formulas (11.21)-(11.23), the argument $\chi^{j p s k}$ is completely arbitrary, we can put $\chi^{j p s k}=\psi^{j p s k}$, in particular. Then Eqs. (11.21)-(11.23) yield that $\mathcal{G}(\psi)=0$. As the next choice, we put $\chi^{j p s k}=\phi^{j p s k}$. Then Eq. (11.13) combined with Eqs. (11.21)-(11.23), yields

$$
\mathcal{G}(\chi)=\mathcal{G}(\phi)+\frac{1}{4 !}\left(O_{1}+O_{2}+O_{3}\right)
$$

It thus remains to compute the $O$ terms. The corresponding calculation is straightforward and simple if we use the representation (11.12). Then we find

$$
\begin{aligned}
O_{1}^{i j k l}(\phi, \psi, \phi) & =O_{2}^{i j k l}(\psi, \phi, \phi) \\
& =O_{3}^{i j k l}(\phi, \phi, \psi) \\
& =8 \frac{\varepsilon}{\mu}{ }^{(i j} \psi^{k|m n| l)} g_{m n}^{\circ} .
\end{aligned}
$$

Note that this result is valid for all possible tensors $\psi$, not only Eq. (11.8) which means that we can further use Eq. (11.25) for the future calculations involving more general nonlinear pieces (in particular, for the case with a nontrivial $m^{i j}$ ). Using then Eq. (11.25) in Eq. (11.24), we get

$$
\left.\mathcal{G}^{i j k l}(\phi+\psi)=-\frac{\varepsilon}{\mu^{2}} \sqrt{-g}^{\circ}{ }^{(i j} g^{1} k l\right) .
$$

Here we denoted

$$
\stackrel{1}{g}^{i j}:=\stackrel{\circ}{g} i j-\frac{\mu}{\sqrt{-g}} \psi^{(i|m n| j)} \stackrel{\circ}{g}_{m n} .
$$

Hence, a purely dielectric nonlinear moving medium will in general exhibit the birefringence effect: the light will propagate in such a medium along the cone of the original optical metric $\stackrel{\circ}{g}^{i j}$ (one may call it ordinary ray), and along the second cone determined by the metric $g^{i j}$ ("extraordinary" ray). 
The explicit form of the extraordinary optical metric is obtained when we substitute Eq. (11.20) into Eq. (11.27):

$$
\begin{aligned}
g^{1 j} & =\stackrel{\circ}{g^{i j}}-\mu P_{k} e^{k(i} u^{j)}+\frac{1}{\varepsilon} u_{k} e^{k(i} P^{j)}, \\
& ={ }^{\circ}{ }^{i j}+\mu e^{k(i} u^{j)} F_{k l} u^{l}-\frac{1}{\varepsilon} u_{k} e^{k(i} F^{j) l} u_{l} .
\end{aligned}
$$

These results generalize the special cases considered in [15].

As an example, let us consider the case of double refraction caused by an electric field. We consider the medium in its rest frame and use adapted coordinates such that $u^{i}=\delta_{0}^{i}$ $=(1, \overrightarrow{0})$. We assume that the dielectric permittivity is given by

$$
\varepsilon=\bar{\varepsilon}+a \mathbf{E}^{2}
$$

Here $\bar{\varepsilon}$ and $a$ are constant parameters. The components of the electric 3-vector $\mathbf{E}$ are defined as usual, $E_{a}=-F_{0 a}$. Then we find

$e^{0 a}=-e^{a 0}=-2 a E^{a}, \quad$ and $\quad P^{j}=u_{i} F^{i j}=\left(0, E^{b}\right)$.

The spatial indices are lowered and raised with the help of the 3-metric $\delta_{a b}$ (we neglect gravity). As a result, from Eq. (11.31) we obtain

$$
P_{k} e^{k i}=\left(-2 a \mathbf{E}^{2}, \mathbf{0}\right), \quad u_{k} e^{k i}=\left(0,-2 a E^{b}\right) .
$$

Finally, using all this in Eq. (11.28) we find the components of the extraordinary optical metric:

$g^{00}=n^{2}\left(1+\frac{2 a}{\varepsilon} \mathbf{E}^{2}\right), \quad g^{a b}=-\delta^{a b}-\frac{2 a}{\varepsilon} E^{a} E^{b}$.

Here $n:=\sqrt{\varepsilon \mu}$ is the refraction index of the medium. In this way we obtain a natural description of the optical Kerr effect (see [28], for example) when birefringence is induced by the applied electric field.

\section{DISCUSSION AND CONCLUSION}

In this paper, we have performed a systematic analysis of the light propagation for two classes of local nonlinear electrodynamics models on the basis of the Fresnel approach. We studied (a) general local nonlinear Lagrangian theories, and (b) moving isotropic nonlinear matter. In the former case, the Lagrangian (3.2) is an arbitrary function of the two electromagnetic invariants, whereas in the latter case, the permeability and permittivity functions of the medium (11.1) depend arbitrarily on the electromagnetic field.

The study of the first class of models reveals the generic nature of the birefringence effect: The quartic Fresnel surface reduces to the two light cones for all nonlinear Lagrangians (3.2). We show that the resulting optical metrics are always real and have the correct Lorentzian signature. In this way, we confirm and extend the recent results of $[14,15]$. Furthermore, we are able to demonstrate the validity of the so-called closure-no birefringence conjecture in the context of nonlinear electrodynamics: Birefringence is absent (and thus the quartic Fresnel surface reduces to a unique light cone) if and only if the effective constitutive (or jump) tensor satisfies the closure property.

The nonlinear isotropic moving matter with the constitutive law (11.1) gives a sound example of a model in which the effective constitutive tensor naturally has nontrivial axion and skewon contributions. Accordingly, one should then expect that the Fresnel surface remains quartic, in general [21]. However, for nonmagnetic material media, we show that birefringence is again the generic effect. The Fresnel surface factorizes into two light cones, one of which corresponds to the Gordon optical metric (independent of nonlinearities), whereas the other [Eq. (11.28)] manifests the nonlinear properties of the model. The optical Kerr effect represents a particular example of our general derivations. It is worthwhile to mention other natural applications of our formalism and, in particular, of the general Fresnel equations (2.10) and (2.11): nonlinear electrodynamics for the case of finite temperature [19] or Casimir [29] quantum vacuum. We will discuss these cases elsewhere.

As a final remark, we feel it is necessary to comment on the recent paper [30] which also discusses wave propagation in local Lagrangian nonlinear electrodynamics. Our results basically agree within the more narrow class of models considered in [30]. However, in our opinion, the concept of birefringence is misused in [30] in the sense that it is applied there also to the case when the Fresnel quartic surface does not factorize into the two light cones. We find this unfortunate because it contradicts the standard terminology of the classical crystal optics. Namely, as it is well known (see [28], for example), an electromagnetic wave falling on a surface of a uniaxial crystal is refracted, giving rise to the two waves, ordinary and extraordinary. Correspondingly, such an effect is called birefringence. The mathematical explanation of this phenomenon is based precisely on the fact that the quartic Fresnel surface decomposes into a product of the two light cones for uniaxial crystal. In contrast, for a biaxial crystal the Fresnel surface does not factorize. As a result, an electromagnetic wave refracts on the surface of a biaxial crystal in a more complicated way giving rise to what is known as the conical refraction which is markedly distinct from the birefringence in a uniaxial crystal. In conformity with this standard terminology, we thus prefer to speak of birefringence, in the broader context of the general local nonlinear models, only when the Fresnel wave surface factorizes into the product of two light cones. This makes the introduction of a notion of "bimetricity" [30] redundant and unnecessary.

\section{ACKNOWLEDGMENTS}

The work of Y.N.O. was partially supported by FAPESP, and G.F.R. would like to thank the German Academic Exchange Service (DAAD) for financial support. 
[1] M. Born and L. Infeld, Proc. R. Soc. London A144, 425 (1934).

[2] W. Heisenberg and H. Euler, Z. Phys. 98, 714 (1936) (in German).

[3] C. Itzykson and J.-B. Zuber, Quantum Field Theory (McGrawHill, New York, 1985).

[4] J.S. Heyl and L. Hernquist, J. Phys. A 30, 6485 (1997).

[5] A.A. Tseytlin, in The Many Faces of the Superworld, Yuri Golfand Memorial Volume, edited by M. Shifman (World Scientific, Singapore, 2000), pp. 417-452.

[6] G.W. Gibbons and D.A. Rasheed, Nucl. Phys. B454, 185 (1995).

[7] R. Kerner, A.L. Barbosa, and D.V. Gal'tsov, in 37th Karpacz Winter School Of Theoretical Physics: New Developments In Fundamental Interactions Theories (Karpacz, Poland, 2001), pp. 377-389.

[8] D.I. Blokhintsev and V.V. Orlov, Zh. Eksp. Teor. Fiz. 25, 513 (1953).

[9] M. Lutzky and J.S. Toll, Phys. Rev. 113, 1649 (1959).

[10] G. Boillat, Ann. Inst. Henri Poincare, Sect. A 5, 217 (1966); G. Boillat, J. Math. Phys. 11, 941 (1970).

[11] J. Plebański, Lectures on Non-Linear Electrodynamics (Nordita, Copenhagen, 1970).

[12] S.A. Gutiérrez, A.L. Dudley, and J.F. Plebanski, J. Math. Phys. 22, 2835 (1981); H.S. Ibarguen, A. Garcia, and J. Plebanski, ibid. 30, 2689 (1989).

[13] S.L. Adler, Ann. Phys. (N.Y.) 67, 599 (1971); Z. BialynickaBirula and I. Bialynicki-Birula, Phys. Rev. D 2, 2341 (1970); W.-Y. Tsai and T. Erber, ibid. 12, 1132 (1975).

[14] M. Novello, V.A. De Lorenci, J.M. Salim, and R. Klippert, Phys. Rev. D 61, 045001 (2000); V.A. De Lorenci, R. Klippert, M. Novello, and J.M. Salim, Phys. Lett. B 482, 134 (2000).

[15] V.A. De Lorenci, and M.A. Souza, Phys. Lett. B 512, 417 (2001); V.A. De Lorenci and R. Klippert, Phys. Rev. D 65 , 064027 (2002).
[16] G.W. Gibbons and C.A.R. Herdeiro, Phys. Rev. D 63, 064006 (2001).

[17] J.P.S. Lemos and R. Kerner, Gravitation Cosmol. 6, 49 (2000).

[18] I.T. Drummond and S.J. Hathrell, Phys. Rev. D 22, 343 (1980); J.I. Latorre, P. Pascual, and R. Tarrach, Nucl. Phys. B437, 60 (1995); G.M. Shore, ibid. B460, 379 (1996).

[19] W. Dittrich and H. Gies, Phys. Rev. D 58, 025004 (1998); H. Gies, ibid. 60, 105033 (1999).

[20] Yu.N. Obukhov, T. Fukui, and G.F. Rubilar, Phys. Rev. D 62, 044050 (2000).

[21] G.F. Rubilar, Yu.N. Obukhov, and F.W. Hehl, Int. J. Mod. Phys. D (to be published); G.F. Rubilar, Ph.D. thesis, University of Cologne, 2002.

[22] F.W. Hehl, Yu.N. Obukhov, and G.F. Rubilar, Int. J. Mod. Phys. A (to be published).

[23] I.E. Tamm, J. Russ. Phys.-Chem. Soc. 57, 209 (1925) (in Russian); [Reprinted in I.E. Tamm, Collected Papers (Nauka, Moscow, 1975), Vol. 1, pp. 33-61 (in Russian)].

[24] W.-T. Ni, "A non-metric theory of gravity," Dept. Physics, Montana State University, Bozeman Report, 1973. The paper is available via http://gravity5.phys. nthu.edu.tw/; W.-T. Ni, Phys. Rev. Lett. 38, 301 (1977).

[25] J.F. Nieves, and P.B. Pal, Phys. Rev. D 39, 652 (1989); J.F. Nieves and P.B. Pal, Am. J. Phys. 62, 207 (1994).

[26] H.F. Kremer, Phys. Rev. 139, B254 (1965); H.F. Kremer, J. Math. Phys. 8, 1197 (1967).

[27] W. Gordon, Ann. Phys. (Leipzig) 4, 421 (1923).

[28] L.D. Landau and E.M. Lifshitz, Electrodynamics of Continuous Media (Pergamon, Oxford, 1960), p. 327, Sec. 80; L.D. Landau, E.M. Lifshitz, and L.P. Pitaevskii, Electrodynamics of Continuous Media, 2nd ed. (Pergamon, Oxford, 1984), p. 347, Sec. 100.

[29] K. Scharnhorst, Phys. Lett. B 236, 354 (1990); G. Barton, ibid. 237, 559 (1990); G. Barton and K. Scharnhorst, J. Phys. A 26, 2037 (1993).

[30] M. Visser, C. Barcelo, and S. Liberati, in the Festschrift of M. Novello, gr-qc/0204017. 